\title{
Excess neurological death in New York City after the emergence of COVID-19
}

\author{
Adam de Havenon $^{1}$ (D) John P. Ney ${ }^{2} \cdot$ Brian Callaghan $^{3} \cdot$ Shadi Yaghi $^{4} \cdot$ Jennifer J. Majersik ${ }^{1}$
}

Received: 16 June 2020 / Revised: 11 July 2020 / Accepted: 14 July 2020 / Published online: 20 July 2020

c) Springer-Verlag GmbH Germany, part of Springer Nature 2020

\section{Dear Sirs,}

New York City (NYC) was the first epicenter of the coronavirus disease 2019 (COVID-19) pandemic in the United States. Beginning in mid-March 2020, the number of deaths in NYC increased above the typical 1000/week, eventually reaching 7726 deaths in the week ending April 11 [1]. That same week, 4441 NYC deaths were attributed to COVID19 [1]. This suggests there were 3285 non-COVID deaths that week, over 2000 of which were excess. These excess non-COVID deaths were attributed to 11 categories, including the main neurological causes of death-cerebrovascular and Alzheimer's disease [2]. We compared the excess non-COVID deaths from cerebrovascular and Alzheimer's disease to other causes of death.

This is a secondary analysis of the National Center for Health Statistics Provisional Death Counts [1]. The cause of death is adjudicated from death certificates using NCHS methodology, but may be later updated to reflect new data [3]. The primary outcome is the excess non-COVID deaths of the eleven NCHS-specified natural causes of death, including cardiac, malignancy, renal, diabetes, influenza/ pneumonia, chronic lower respiratory disease, other respiratory, sepsis, other/undetermined, Alzheimer's disease, and cerebrovascular in NYC for the weeks ending 01/04/20-06/20/20. Eighteen data points were missing, in which case we carried forward the prior value. Because the first excess non-COVID deaths were recorded in the week

Adam de Havenon

adam.dehavenon@hsc.utah.edu

1 Department of Neurology, University of Utah, $175 \mathrm{~N}$. Medical Dr., Salt Lake City, UT 84132, USA

2 Department of Neurology, Boston University, Boston, USA

3 Department of Neurology, University of Michigan, Ann Arbor, USA

4 Department of Neurology, New York University, New York City, USA ending $03 / 21 / 20$, we stratified by 11 -week periods ending $01 / 04 / 20-03 / 14 / 20$ and $03 / 21 / 20-05 / 30 / 20$, to calculate percentage change and test for differences using Student's $t$ test.

For $01 / 04 / 20-03 / 14 / 20$, the average weekly number of natural deaths in NYC was 1101 (range 1049-1142), while for 03/21/20-05/30/20, the average was 3247 (range $1128-7682)$, a $195 \%$ increase $(p<0.001)$ (Fig. 1a). Figure 1a shows excess deaths return to a near normal level after 05/30/20. During 03/21/20-05/30/20, COVID-19 deaths averaged 1578 a week, failing to account for the entirety of excess death. Figure $1 \mathrm{~b}$ shows the concept of excess non-COVID deaths, which averaged 1670/week during 03/21/20-05/30/20.

The causes of non-COVID excess death are shown in Fig. 1c, illustrating a prominent increase in cardiac death, which was the most common non-COVID excess cause of death. An interactive figure of all the COVID-19 and nonCOVID excess deaths is at https://www.icloud.com/pages /0vW0yM-3-PwN8m3IAR6F5DTHQ\#Figure_1D. The percentage changes are in Fig. 1e. Deaths attributed to cerebrovascular and Alzheimer's disease increased $11.8 \%$ and $38.9 \%$, respectively, from an average of 41.2 to 49.0 a week for cerebrovascular $(p=0.194)$ and 21.3 to 29.6 a week for Alzheimer's $(p=0.126)$.

In mid-March 2020, after the rise in COVID-19 infections in NYC, excess non-COVID deaths increased for cerebrovascular and Alzheimer's disease, but this increase was far less than multiple other causes of death. Lack of widespread COVID-19 testing during this period [4] means that many of the excess non-COVID deaths were likely due to complications from undiagnosed COVID-19. While neurologic complications have been reported with COVID-19 infection, they are not as common as other organ systems [5, 6]. The relatively small $11.8 \%$ increase in cerebrovascular death suggests that while stroke may complicate COVID-19 infection, it may not be as fatal as other complications. The larger $38.9 \%$ increase in Alzheimer's deaths may reflect their goals of care or older age $[5,6]$. The other possible explanation 


\section{A}

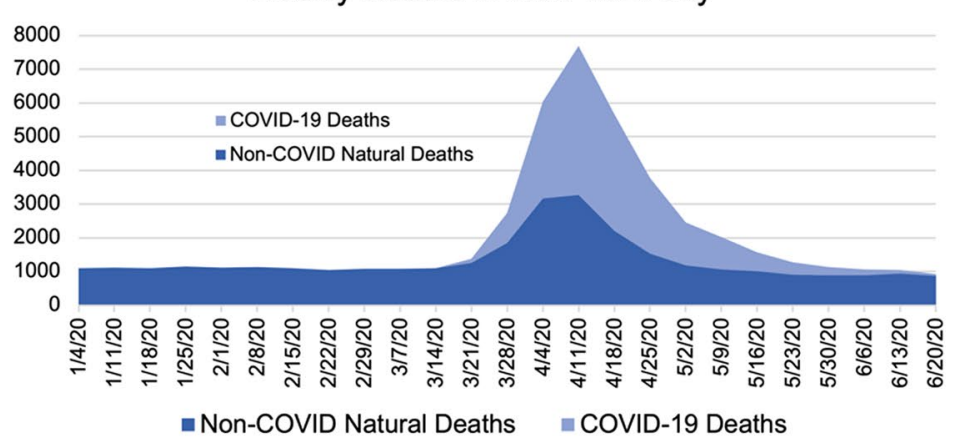

C

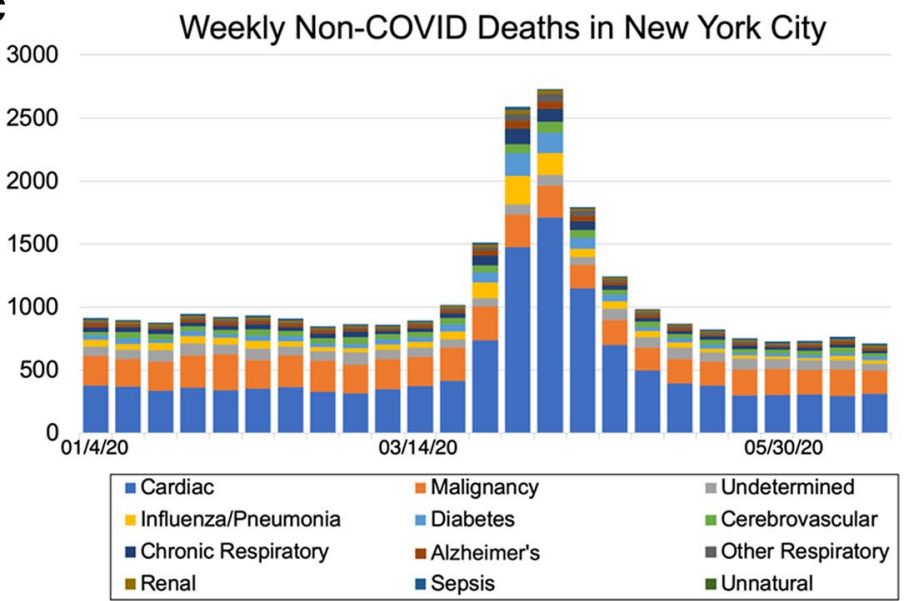

B

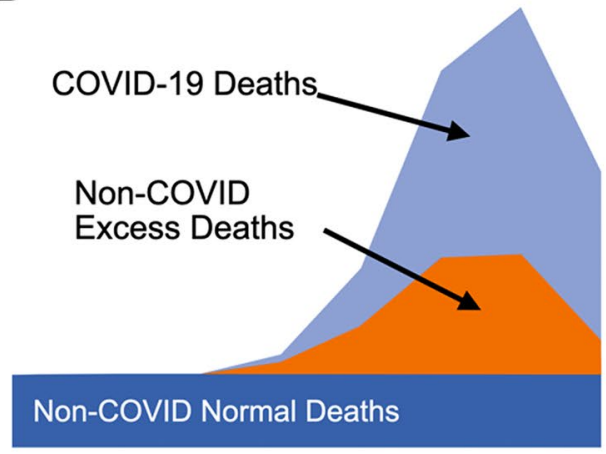

E

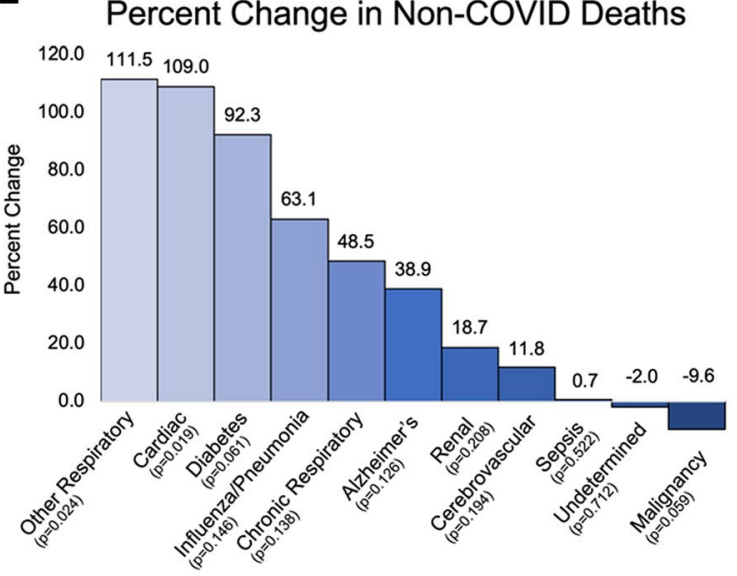

Fig. 1 Weekly natural deaths in NYC between the weeks ending 01/04/20 and 06/20/20. a All natural deaths, with COVID-19 deaths shown in light blue. b Illustration of the excess death (orange) attributed to non-COVID causes. c The weekly counts of deaths from the 11 non-COVID causes. $\mathbf{d}$ Interactive figure of the weekly death counts: https://www.icloud.com/pages/0vW0yM-3-PwN8m3IAR6 F5DTHQ\#Figure_1D. e Percentage change in the non-COVID causes of death, comparing the weeks ending 03/21/20-05/30/20 to the weeks ending 01/04/20-03/14/20 for the excess non-COVID deaths is that there was reduced access to healthcare during the pandemic in NYC.

The main limitation of our study is that NCHS cause of death may not reliably identify the underlying cause of death, particularly in the absence of widespread COVID-19 testing. These data are also provisional and are subject to revision. Despite these limitations, we found that the two most common neurological causes of death, cerebrovascular and Alzheimer's disease, increased comparatively less than pulmonary, cardiac, and diabetic deaths in NYC during the recent peak of COVID-19 mortality.

Funding Dr. de Havenon is supported by NIH-NINDS K23NS105924.

\section{Compliance with ethical standards}

Conflicts of interest Dr. de Havenon has received investigator initiated funding from AMAG and Regeneron pharmaceuticals. Dr. Callaghan consults for a PCORI grant, DynaMed, and performs medical legal consultations including consultations for the Vaccine Injury Compensation Program. Dr. Majersik reports NIH/NINDS funding U24NS107228, funding for Associate Editor at Stroke, consulting fees for Foldax scientific advisory board, and is an Editorial Board member of Neurology. The remaining authors report no potential conflicts of interest.

Ethical approval IRB approval was not required for this retrospective analysis of deidentified data per the University of Utah Institutional Review Board Guidelines.

Availability of data The data are publicly available from the Centers for Disease Control. 


\section{References}

1. Provisional Death Counts for Coronavirus Disease (COVID-19) (2020) https://www.cdc.gov/nchs/nvss/vsrr/covid19/tech_notes .htm. Accessed 26 May 2020

2. Johnson CO, Nguyen M, Roth GA et al (2019) Global, regional, and national burden of stroke, 1990-2016: a systematic analysis for the Global Burden of Disease Study 2016. Lancet Neurol 18:439-458. https://doi.org/10.1016/S1474-4422(19)30034-1

3. Instruction Manuals (2019) https://www.cdc.gov/nchs/nvss/instr uction_manuals.htm. Accessed 04 May 2020
4. Sharfstein JM, Becker SJ, Mello MM (2020) Diagnostic testing for the novel coronavirus. JAMA 323:1437-1438. https://doi. org/10.1001/jama.2020.3864

5. Coronavirus Disease 2019 (COVID-19): Complications. https:// bestpractice.bmj.com/topics/en-us/3000168/complications

6. Richardson S, Hirsch JS, Narasimhan M et al (2020) Presenting characteristics, comorbidities, and outcomes among 5700 patients hospitalized with COVID-19 in the New York City area. JAMA. https://doi.org/10.1001/jama.2020.6775 